\title{
LES SOUS-REGIONS RURALES DE LA PROVINCE ORIENTALE DANS LA DYNAMIQUE DE L'HISTOIRE RECENTE DU ZAIRE: DE 1960 à 1985 (CAS DU BAS-UÉLÉ)
}

\section{MILY DENDA-SAKALA, Dieudonné}

Avenue Regideso 50

Buta

République Démocratique du Congo

\section{SUMMARY}

THE RURAL SUB-REGIONS OF THE EAST-PROVINCE IN THE RECENT HISTORICAL DYNAMICS OF ZAÏRE: FROM 1960 TO 1985 (THE CASE OF THE BAS-UÉLÉ)

This study takes the Sub-Region of the Bas-Uléle as an example of the recent history of the rural areas in Zaire. It gives a description from an insider's point of view based on local research, results in a testimony relating the complete deterioration of rural potentialities in Zaire.

The article describes how in the first years after independence, the rich natural resources and the colonial infrastructure brought prosperous development. From 1964 to 1966, however, the Simba-rebellion ravaged the area, destroying a great deal of the infrastructure and caused large-scale massacres among the local population. 
After the crushing of the rebellion, the subsequent revival was quickly halted by constant bureaucratic hindrances and interference from Mobutu's MPR. "Zairisation" led to further deterioration of the economic infrastructure.

From 1980 on, the Sub-Region was neglected more and more by the central government and even by its elected representatives. Finally, the liberalising of goldprospecting withdrew farmers, schoolchildren and teachers form their normal activities leading only to more misery in the area.

KEY WORDS: Development, Local Administration, Politics, Zaire

\section{INTRODUCTION}

L'histoire du Zaire est étalée dans une abondante littérature. C'est une histoire bien connue, mais elle est loin d'être complète. La tache des intellectuels zaïrois demeure gigantesque, impérieuse et urgente, disait un éminent professeur. Lumumba, quant à lui, avait affirmé que l'Afrique ré-écrira sa propre histoire. ${ }^{1}$ Cette ambitieuse tache ne pourrait se réaliser que lorsque chacun apporte sa pierre à l'édifice. C'est dans cette logique que se circonscrit la présente étude qui se veut une modeste contribution à la connaissance et à la réécriture de l'histoire du Zaïre dans une optique de l'évolution récente des différentes sous-régions rurales. Dans ce pays aux dimensions continentales, les centres urbains sont opposés aux milieux ruraux. Depuis l'époque coloniale cette stratification continue de présenter la dichotomie entre différentes entités politico-administratives. Plusieurs observations scientifiques établissent des conclusions selon lesquelles les centres urbains font régulièrement objets de diverses études pluridisciplinaires au détriment des régions rurales qui, elles, sont souvent reléguées dans les oubliettes.

Dans le présent article, nous avons opté pour la région rurale du Bas-Uélé comme cible de notre analyse. Deux raisons majeures ont guidé ce choix. Primo, le

'TCHIMANGA wa TCHIBANGU, Histoire du Zaire, CERUKI, 1969. 
Bas-Uélé constitue un meilleur exemple de sous-régions rurales les plus éloignées de la capitale du pays. Secundo, dans l'abondante littérature dont nous avions fait allusion plus haut, le Bas-Uélé demeure le plus grand absent.

A cet effet, nous nous faisons l'obligation de briser ce silence et parvenir ainsi à faire entendre, tant soit peu, la voix du Bas-Uélé. Par mesure de concision, nous circonscrivons l'étude entre 1960 et 1985 . Cette limitation voulue, consiste à débuter l'étude à partir de l'accession du pays à la souveraineté nationale. Elle fixe la fin de la période observée en 1985, année proclamée par le Président Mobutu comme étant le début d'un nouveau septennat social. Pendant un quart de siècle, quel a été le parcours des sous-régions rurales en général, et celui du Bas-Uélé en particulier.

Le Bas-Uélé est une des sous-régions de la région du Haut-Zaire. Comme toutes les autres sous-régions rurales du Zaire, le Bas-Uélé a traversé et traverse encore en ces jours une situation dont l'étude ne pourrait se faire en quelques pages d'un article, tellement qu'il y a des choses à dire. Cependant, il demeure nécessaire et indispensable, voire impérieux, de produire un matériel de base afin de jalonner les pistes pouvant faciliter des recherches ultérieures sur cette sous-région.

La présente réflexion est le fruit de longues années de recherche menée à Buta, Kisangani et ailleurs. C'est, en outre, un chapitre d'un mémoire que nous avons présenté pour le grade de Master en Gestion de Développement [Ruca-Anvers]. Le présent texte obéit à une approche systémique qui prétend aborder de façon globale le Bas-Uélé dans son ensemble.

Le sujet sera traité en trois épisodes. Le premier parlera du Bas-Uélé de 1960 à 1965, le deuxième épinglera la situation du Bas-Uélé de 1965 à 1973. Enfin, le troisième portera sur la période déterminante de 1973 à 1985. Avant d'aborder l'étude de ces trois périodes susmentionnées, nous présenterons un bref aperçu de la sous-région avant 1960, en même temps que nous rassemblerons une vue globale sur l'environnement et les ressources du milieu en étude.

\section{A. Bref aperçu du Bas-Uélé avant 1960}

Apres plusieurs essais tentatives et remaniements du Ministère de Colonie et du Gouvernorat général du Congo Belge, le district du Bas-Uélé fut créé en 1912 lorsque l'Arrêté Royal du 28 mars 1912 (B.O., pp. 356-369) fut publié. ${ }^{2}$ Il est

\footnotetext{
${ }^{2}$ L. de St. MOULIN, "Organisation administrative du Zaïre de 1888 à 1992" in Zaüre-Afrique, $\mathrm{n}^{\circ}$ 261, janvier 1992, Kinshasa.
} 
l'une des cinq sous-régions qui forment la région du Haut-Zaïre. Avant 1960 et quelques années après l'accession du pays à l'indépendance, le Bas-Uélé était le " grenier" de la population de la ville de Kisangani car les abondantes productions vivrières du district étaient acheminées dans cette ville.

Ayant bénéficié de l'économie monétaire et des cultures d'exportation, le district était prospère. C'est dans ce district que l'agriculture avait produit des richesses en coton, en caoutchouc et en produits palmistes estimées en milliers de tonnes. ${ }^{3}$

Les sociétés agricoles qui ont encadré la population du Bas-Uélé étaient entre autres:

- la C.V.C. (Société des Chemins de Fer Vicinaux du Congo)'; - la Comuélé (Compagnie de l'Uélé);

- la Cotonco (Compagnie Cotonnière du Congo);

- la Comaco (Compagnie Agricole et du Commerce).

Du côté socio-culturel, les missionnaires étaient les seuls organisateurs des écoles où étaient formés des enseignants, des agents auxiliaires de l'administration, des menuisiers, des mécaniciens et divers autres cadres comme des moniteurs agronomes, etc. Cette prospérité du Bas-Uélé a continué son chemin jusqu'aux troubles de 1964, tel que nous le verrons dans la suite de notre étude.

\section{B. Environnement et ressources}

\section{Elément géographique et écologique}

Le Bas-Uélé est situé au Nord-Ouest de la région du Haut-Zaïre. Cette sousrégion est caractérisée par un relief progressif allant des plaines aux bas-plateaux. L'altitude moyenne est de plus ou moins $600 \mathrm{~m}^{5}$

\section{a. Le climat}

${ }^{3}$ CHALUX, -, Un an au Congo, Albert Dewit, Bruxelles, 1925, p. 668.

${ }^{4}$ c.v.c.. Congo-Nil, Van Assche. Bruxelles, 1936, p. 17.

${ }^{5}$ F.Z. KAMA., Géographie du Zaire, Paris, Hatier, 1970, p. 11. 
Il est tropical. En période chaude les températures varient entre $25^{\circ} \mathrm{C}$ et $30^{\circ} \mathrm{C}$. En période froide, elles varient entre $18^{\circ} \mathrm{C}$ et $20^{\circ} \mathrm{C}$. Les pluies commencent du début avril jusqu' à la fin juillet; elles reprennent pendant trois mois: octobre novembre - décembre. Il y a donc deux saisons pluvieuses: une grande et une petite. Ces pluies atteignent 1,6 m d'eau. ${ }^{6} \mathrm{La}$ saison sèche, quant à elle, va du mois de janvier au mois d'avril.

\section{b. La flore}

Elle présente une double physionomie, à savoir, la végétation fourrière qui constitue un large domaine allant de l'Itimbiri-Rubi à Likati. Le deuxième domaine est couvert de la savane boisée au niveau des bas-plateaux, couvrant toute la zone de Bondo.

\section{c. L'hydrographie}

Celle-ci regorge un nombre impressionnant de cours d'eau. Les plus importants sont: la Rubi; l'Uélé; la Bamokandi; la Likati; la Gangu-Bili.

Notons en passant que la Likati et la Rubi-Itimbiri avaient joué un rôle important dans la navigation pendaht la colonisation.?

\section{d. Le réseau routier}

Ce vaste réseau du Bas-Uélé doit son existence à la C.V.C. et à la Cotonco. Il comporte des axes suivants: - Aketi-Bunduki-Bumba; Aketi-Buta-Paulis (Isiro); AketiButa-Kisangani; Aketi-Bondo-Bili; Aketi-Bondo-Baye; Akedi-Bondo-Ndu (voir carte l).

\section{e. Le réseau ferroviaire}

Jusqu'en 1975, ce réseau comprenait trois axes principaux:

1. Aketi-Komba-Buta-Paulis-Mungbere;

2. Aketi-Komba-Buta-Titule;

3. Aketi-Komba-Likati-Bondo.

A partir de l'année 1975, un quatrième axe fut inauguré de Aketi à Bumba.

Une observation mérite d'être faite ici. En fait, comme l'on peut constater, le centre de Aketi constituait la plaque tournante de l'économie du Bas-Uélé. La

\footnotetext{
${ }^{6}$ DENIS et WILMET, L'Afrique Centrale et Orientale, PUF, Paris, 1971, p. 117.

${ }^{7}$ CHALUX. op. cit., p. 662.
} 
Direction générale de la C.V.C. y avait son siège. En outre un petit port y était construit et géré par 1'OTRACO. ${ }^{8}$ Quelques bateaux à barges de capacités moyennes desservaient Aketi-Bumba et assuraient ainsi le transport des marchandises en provenance de toutes les zones agricoles de la sous-région. ${ }^{9}$

Concernant le réseau ferroviaire, nous signalons que l'écartement des rails n'était que de $60 \mathrm{~cm}$; cet état de choses rendait les trains trop lents. Tel est l'aspect environnemental du Bas-Uélé. Poursuivant notre observation, nous ajoutons que la structure dont nous venons de parler n'appartient plus qu'à l'histoire, car tout a été démantelé.

\section{Les ressources}

\section{a. Ressources matérielles}

Les ressources matérielles du Bas-Uélé se constituent de sa foret, de son sous-sol et de ses cours d'eau.

\section{${ }^{*} \underline{\text { La forêt }}$}

Lorsque nous évoquons la forêt, nous entendons mentionner trois aspects suivants: l'agriculture, la faune et les essences.

L'agriculture constitue la ressource principale de la sous-région. Elle fournit les diverses cultures vivières ou industrielles qui assurent la subsistance et les revenus de la population. Les plus importantes cultures sont: le manioc, le riz, le coton, le café et les différentes sortes de légumes et fruits.

La faune est constituée d'une grande diversité d'espèces animales. La forêt est très giboyeuse et la chasse y est pratiquée toute l'année. Mais la réglementation coloniale a laissé une loi prohibant la chasse entre août et novembre, période caractérisée par la gestation et la mise bas. Une autre loi avait interdit tout braconnage. Cependant mentionnons tout de suite qu'une chose c'est la loi, une autre en est l'application. Au Zaïre, l'application de cette loi contre le braconnage fut marginalisée et boycottée depuis bientôt 20 ans.

Les essences: la forêt tropicale du Bas-Uélé dispose d'une grande variété d'essences de bois qui, malheureusement, ne connaissent qu'une minime exploitation. Le bois reste une richesse inexploitée. On cite par exemple le bois

\footnotetext{
${ }^{8}$ ONATRA: Office National de Transports qui a remplacé OTRACO: Office de Transport Colonial. cfr. CORNEVIN, p. 307).

${ }^{9}$ C.V.C., op.cit., p. 386.
} 
blanc, le bois noir, le bois rouge et d'autres espèces appréciées pour la production des planches. Une exploitation rationnelle du bois servirait à la fourniture des matériaux de construction de ponts, de maisons, de carrosseries, de bateaux et autres ustensiles.

A part toutes ces ressources mentionnées en provenance de la foret, sont également recensées les plantes médicinales dont l'exploitation reste loin d'être organisée.

\section{* Le sous-sol}

Lorsqu'on observe les cartes économiques du Zaïre produites à l'époque coloniale, on ne se rend compte que des richesses agricoles, limitant les richesses du sous-sol au seul gisement de fer. Depuis la même période l'or était exploité à Baye, une région située dans la zone de Bondo à plus de $580 \mathrm{~km}$ de Kisangani. Depuis l'année 1982, une loi promulguée sur l'exploitation artisanale de l'or et du diamant a révélé que le sous-sol du Bas-Uélé est riche en gisements d'or et de diamant. ${ }^{10}$ Actuellement les sites et carrières d'extraction abondent. L'or et le diamant circulent. L'achat se fait anarchiquement et par des pirates, sous l'oeil désarmé de l'autorité politico-administrative corrompue et défaillante. Il y a une grave crise de gestion.

\section{b. Les ressources humaines}

Le Bas-Uélé compte parmi les régions à faible densité de population. Les quelques agglomérations à forte concentration démographique de la sous-région sont Aketi, Ango, Bambesa, Bondo et Buta. En dehors de celles-ci, la population habite soit le long des routes, des chemins de fer ou simplement au bord des cours d'eau. Le recensement de 1987 nous donne les chiffres de 627.083 personnes, soit $12,3 \%$ de la population totale du Haut-Zaïre. La densité est de 4,2 habitants $/ \mathrm{km}^{2}$. La superficie s'élève à $148.331 \mathrm{~km}^{2}{ }^{11}$ Le même document fait état d'une des causes de faible densité qui est une stérilité pathologique. ${ }^{12}$ Deux grands embranchements ethniques constituent l'essentiel de la population. Il y a d'une part la souche soudanaise qui comprend les Bazande et les Bangbandi. On y ajoute les Bakere. Ceux-ci habitent Ango, Bondo, Buta, Likati, Poko, etc.

\footnotetext{
${ }^{10}$ Nous en parlerons au moment opportun.

${ }^{11}$ GONIDEC, E.P.C., Série Afrique: le Zaïre, Berger Levrault, Paris, 1973, p. 35.

${ }^{12}$ A.I. GONDO, "Chiffres de population ....: les élections législatives de 1987", Zaire-Afrique, $\mathrm{n}^{\circ}$ 264, avril 1992, CEPAS, Kinshasa, p. 229.
} 


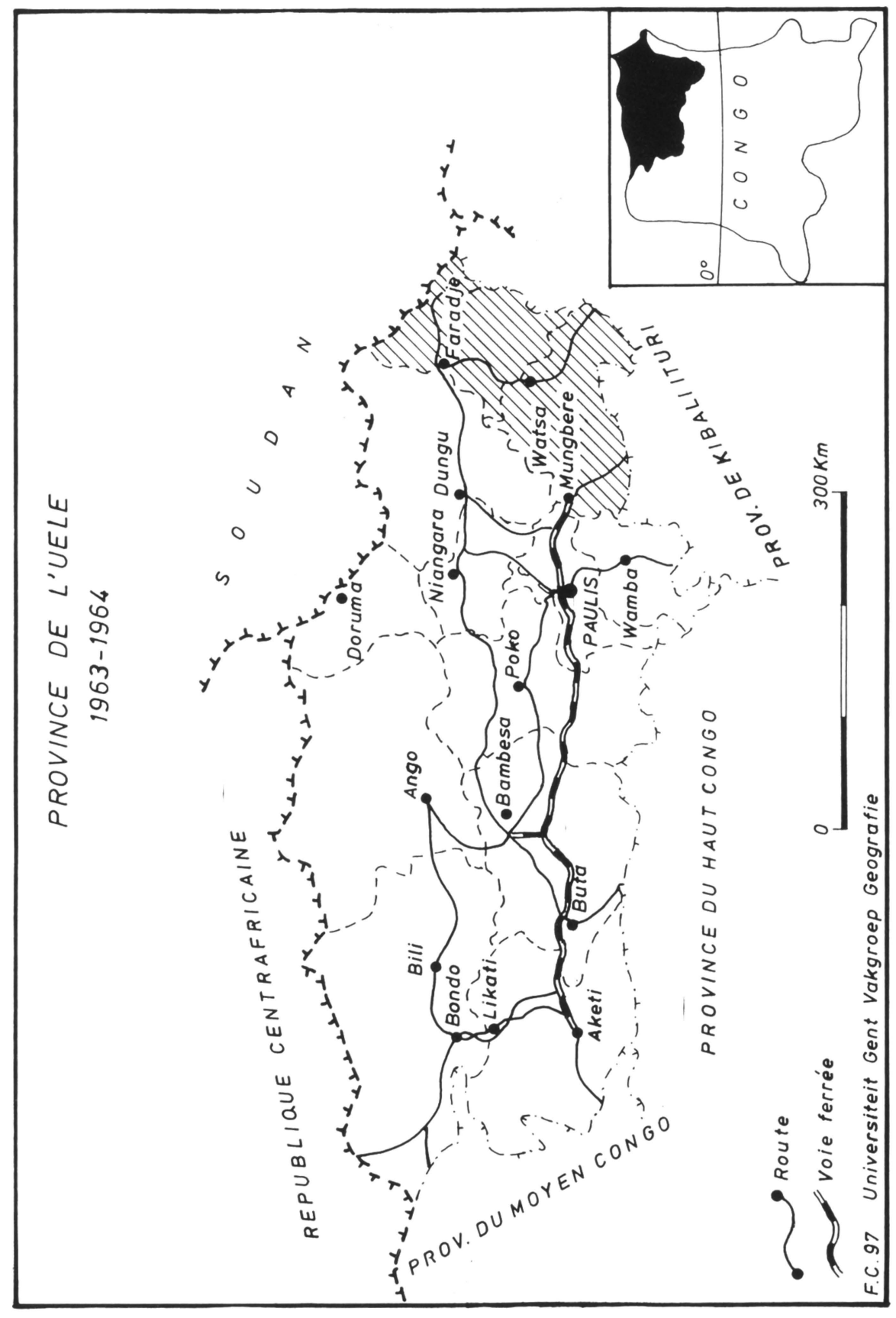


D'autre part, se remarque la souche bantoue composée des Babenge, Babowa, Babenza, qui semblent à mon avis minoritaires. Ils habitent en symbiose avec les Soudanis à Bondo, à Likati et à Buta, sauf à Aketi or l'on ne rencontre que la population bantoue.

Quant en ce qui concerne la subsistance, tous ces groupes ethniques exercent les mêmes activités économiques, à savoir l'agriculture, la chasse et la pèche, prises dans l'ordre décroissant de leur importance.

\section{La structure politico-administrative}

La sous-région de Bas-Uélé est subdivisée en six zones suivantes: Aketi, Ango, Bambesa, Bondo, Buta et Poko. Le chef-lieu a son siège à Buta où sont concentrés les bureaux sous-régionaux de toutes les activités politico-administratives. Le parquet y fonctionne sous l'égide d'un procureur-général, une succursale de la Banque Nationale y fonctionne depuis l'époque coloniale. Le Commissaire sous-régional est le numéro un, il supervise toutes les activités secondé par quatre Assistants chargés respectivement du secteur politique, administratif, culturel et social.

Le Bas-Uélé compte cinquante-deux collectivités dirigées par des chefs de collectivité qui sont tous des chefs coutumiers. Ils sont de fait agents de l'administration. Cette vue globale du Bas-Uélé sert à fixer le lecteur. A présent nous allons aborder l'évolution proprement dite.

\section{Organisation et évolution du Bas-Uélé}

\section{Le Bas-Uélé de 1960 à 1965}

\section{$1^{\circ}$. De 1960 à 1963}

Epargnée de toutes les perturbations socio-politiques jusqu'à la fin du ler semestre de l'année $1964^{13}$, la sous-région alors district du Bas-Uélé vue dans sa globalité était prospère. Christian le confirme en écrivant: En 1963, l'organisation de l économie moderne en Uélé présentait un réseau remarquablement dense de

\footnotetext{
${ }^{13} \mathrm{C}$. COMELIAU, Fonctions économiques et pouvoir politique. La Province de l'Uélé en 1963-1964, Coll. Éd. économiques, $n^{\circ}$ 1, Rép. du Congo p. 9.
} 
voies de communication, $d^{\prime}$ usines agricoles, de plantations et d'installations commerciales. ${ }^{14}$ Le Bas-Uélé a pu réellement profiter de l'agriculture intensive des plantations pour compléter l'agriculture extensive.

Les activités économiques tournaient à plein rendement. La stabilité socio-économique a été la base du bon fonctionnement de l'administration territoriale. Un autre élément circonstanciel très important demeure qu'entre 1960 et 1963 l'infrastructure héritée de la colonisation était encore impeccable.

Autre chose, les expatriés qui évoluaient dans le secteur d'activités économiques ne sont pas rentrés en Europe après 1960. Ceux-ci s'occupaient de différentes branches comme les plantations dites européennes, le traitement d'huile de palme et d'arachide, le traitement du coton, les mines d'or, des ateliers de construction, les transports, le commerce et divers services. Le mérite de toutes ces activités est qu'elles garantissaient le revenu (salaire) à la population autochtone. Pour une meilleure coordination, des sociétés étaient mises en place. Parlons de quelques-unes.

Belgika: une société belge assurait l'encadrement des cultivateurs et l'achat des produits agricoles. Belgika représentait les usines Ford à Buta avec un garage et un atelier pour véhicules.

Sedec-Motor: s'occupait d'un atelier de montage de bicyclettes (marque Modidi) à Buta.

Interfina: Elle assurait la vente des vêtements pour femmes et d'autres produits manufacturés, produits d'alimentation. Elle assurait également la distribution de quincaillerie.

Nogueira et la Maison hollandaise sont aussi citées aux cotés de COTONCO et de COMUELE. ${ }^{15}$

Cotonco: dont on a déjà parlé, avait également sa place.

C.V.C.: nous avons longuement parlé des activités économiques de la CVC, notamment les transports routier, ferroviaire des biens et des personnes, etc.

La C.V.C. a poussé plus loin. Sur le plan social, elle avait construit des logements pour ses travailleurs. Deux hôpitaux de grande importance comptant plus de 500 lits, furent construits, l'un à Aketi et l'autre à Buta. Une école de commis avait

${ }^{14}$ Idem, p. 13

${ }^{15}$ CARBONELLE et KIASCHEM. L'économie des deux Uélés, CEMUBAC, Bruxelles, 1961. 
fonctionné jusqu'en 1975 à Aketi, puis transférée à Isiro (Paulis) avant de fermer ses portes dans les Uélés au profit de Lubumbashi. ${ }^{16}$

Grosso-modo, disons que le Bas-Uélé a prospéré sur le plan socio-économicoculturel, entre 1960 et 1964, grâce aux sociétés privées et aux missions religieuses. Cependant, l'année 1964 avait malheureusement instauré une triste période.

$2^{\circ}$. A partir de 1964: le Bas-Uélé dans les troubles

Jeudi le 6 août 1964, la sous-région du Bas-Uélé, érigée entre-temps en province de l'Uélé s'est effondrée avec la rébellion. ${ }^{17}$ Dès lors, le Bas-Uélé vécut dans une précarité et une insécurité indescriptible. Cette triste période s'est étendue de 1964 à 1966.

Le Bas-Uélé est une des sous-régions qui avaient été atteintes de plein fouet par la rébellion qui avait sévi dans la province orientale. La rébellion avait pénétré dans le Bas-Uélé avec une extrême facilité dont les explications prennent une connotation souvent légendaire. En effet, les éléments de l'armée régulière congolaise, basés à Gombari, à Buta et à Aketi, pris de panique généralisée n'ont pu opposer aucune résistance à 1'APL sinon se convertir en rebelles lorsqu'ils étaient repérés et appréhendés. Ces bataillons mis en débandade, avaient abandonné tout le matériel militaire dont ils disposaient à la portée des troupes rebelles. Ayant récupéré ledit matériel, l'APL, sous le commandement de Gbenye Christophe ${ }^{18}$ qui entendait faire du Bas-Uélé le bastion de sa politique, s'en est servi pour assiéger la sous-région. Gbenye, poussé par ses ambitions obscures, a d'abord lui-même dirigé les opérations de ravages à Aketi, Ango et Buta, avant de passer la commande à des généraux et colonels comme Andelekani, Makondo ${ }^{19}$, Ngbatala, Olombe, pour continuer dans les autres directions, notamment à Bondo, à Isiro et ont même poussé jusque Yakoma dans la province de l'Equateur. Dans le présent article, le terme "opérations" exprime des massacres, des assassinats, des tueries et des casses qui ont été anarchiquement infligés à la sous-région. Les exactions de rebelles-Simba ${ }^{20}{ }^{21}$ accompagnées de brutalités sanguinaires ne peuvent être mesurées que par des dégâts matériels et les pertes humaines enregistrés dont les conséquences demeurent encore d'actualité aujourd'hui. De façon globale ces dégâts pourront être présentés de manière suivante.

\footnotetext{
${ }^{16}$ Elisabethville au Shaba (Katanga).

${ }_{17}^{17}$ J. VERHOEVEN, Dagboek, Gijzelaars over Makondo, Diest, 1965, p. 15.

${ }^{18}$ Ancien Président de la République Populaire du Congo de Stanleyville.

${ }^{19}$ On l'appelait "l'homme seul", Jan Verhoeven, op.cit. p. 196.

${ }^{20}$ Simba désigne lion en Swahili et signifie fort, sans peur, indomptable.

${ }^{21}$ B. VERHAEGEN, Rébellion au Congo Maniema, Tome II, CRISP. 1969, p. 345
} 


\section{a. Dégâts matériels}

Il est important de signaler ici que la rébellion a mis en carton toute l'oeuvre de la colonisation. Les structures organisationnelles qui ont fait la stabilité et la prospérité socio-économiques du Bas-Uélé furent détruites complètement. Ce fut le début de la dégradation de l'écosystème de la sous-région toute entière.

Concrètement, toutes les institutions, tous les édifices ont été systématiquement pillés avant d'être saccagés par les rebelles-Simba. Ce furent notamment les églises, les écoles, les habitations qui avaient appartenu aux "évolués". Les magasins, et même les hôpitaux ne furent guère épargnés. Pire encore, toutes les archives furent simplement et purement brûlées. Pour justifier ces mésaventures, les chefs rebelles (Gbenye) avaient choisi le mensonge et les promesses fallacieuses du genre: tous ceux qui recevraient Mulele et ses hommes de bon coeur seraient grands et riches ... afin d'asseoir leur anarchie.

Toujours à propos des exactions, toutes les usines et ateliers installés dans la sous-région furent détruits. D'après un ancien Simba, seules les centrales électriques de Dingila dans la zone de Bambesa et d'Aketi étaient épargnées afin de continuer de fournir l'électricité. En effet, pour les chefs rebelles, Dingila et Aketi constituaient deux centres stratégiques. ${ }^{22}$

Outre les édifices, toutes les infrastructures de transport et sanitaires avaient été confisquées, occupées et enfin détruites par les rebelles. Les débuts de l'année 1965, les destructions se sont accélérées et amplifiées, car les Simba ayant perdu leur immunité, redoutaient les représailles de l'ANC (Armée Nationale Congolaise). Et ne disposant plus ni de moyens matériels, ni d'autres stratégies, les Simba ont du recourir à la destruction des ponts pour empêcher l'ANC de les atteindre. Tous ces actes de barbarie et de vandalisme avaient concouru à isoler la sous-région, à la fois coupée de Kisangani, de Isiro et de Bumba. Le Bas-Uélé avait ressemblé à une jungle où les seuls maîtres étaient les Simba. Rien n'avait été épargné. Bétails, volailles, habitations, édifices, etc, rien n' avait plus appartenu à personne. Sans lois, ni règles, la seule volonté des responsables de l'APL dictait le comportement social. L'insécurité et la panique pouvaient se vérifier par le fait que les cités étaient désertées. La population habitait dans la brousse où elle s'était retranchée. Les cités ne reprenaient l'ambiance que lorsqu'un appel exprès était formulé pour un meeting, une communication ou simplement pour des exécutions publiques de victimes tombées entre les mains de rebelles ${ }^{23}$ tel que nous allons le voir au point suivant.

\footnotetext{
${ }^{22}$ A. DOMBA. un ancien lieutenant-Simba interrogé à Buta, le 19 octobre 1991 à 1'ISP/Buta.

${ }^{23}$ D. MILY, Témoignage personnel dun garçon de 11 ans en 1965 à Aketi et à Likati.
} 


\section{b. Les pertes humaines}

Le Bas-Uélé se souviendra encore très longtemps des pertes humaines enregistrées pendant la rébellion. Plusieurs personnes interrogées sont unanimes et affirment que Gbenye lui-merrre avait recommandé de tuer tout collaborateur du Blanc, quel qu'il fut. Les personnes visées étant principalement les intellectuels et les membres des autres formations politiques que le MNC. Les massacres étaient dirigés vers les commis (clercs), les enseignants et les missionnaires. Les premières victimes ayant été surprises furent le Commissaire de district et les six administrateurs territoriaux et leurs commis. Tous les chefs de région et souschefs coutumiers avaient été tués. Des enseignants avaient été appréhendés, jugés et exécutés publiquement. Parmi les victimes 24 missionnaires dont une soeur furent arrêtés et tués à Buta le 30 mai $1965^{24}$ queque temps avant l'exécution de la Béate Anualité à Isiro. Quelques chiffres pouvant illustrer l'ampleur des massacres ont été rassemblés par le Bureau du diocèse de Buta à l'Evêché dans un rapport dont voici l'extrait.

Tableau $\mathbf{n}^{\circ} 1$

\begin{tabular}{|l|l|l|l|l|l|l|l|l|l|}
\hline Statut & $\begin{array}{l}\text { Nombre } \\
\text { Aketi }\end{array}$ & $\begin{array}{l}\text { /territoire } \\
\text { Ango }\end{array}$ & Bambera & Bondo & Buta & Poko & M. & F \\
\hline Enseignant & 249 & 133 & 146 & 250 & 266 & 122 & 1148 & 1 & $\begin{array}{l}2221 \\
*\end{array}$ \\
\hline Clercs & 92 & 76 & 53 & 22 & 102 & 39 & 384 & - & \\
\hline $\begin{array}{l}\text { Missionai- } \\
\text { res }\end{array}$ & $?$ & $?$ & $?$ & $?$ & 24 & $?$ & 23 & 1 & \\
\hline $\begin{array}{l}\text { Chef } \\
\text { coutumiers } \\
\text { Simba }\end{array}$ & 8 & 4 & 9 & 9 & 7 & 13 & 50 & - & \\
\hline Suspectés & 197 & - & 152 & - & 222 & 26 & 597 & - & \\
\hline Autres & $?$ & $?$ & $?$ & $?$ & $?$ & $?$ & $?$ & $?$ & $\begin{array}{l}\text { inno- } \\
\text { mera- } \\
\text { bles }\end{array}$ \\
\hline
\end{tabular}

*Observation: Parmis ces 2221 tués, 1166 enseignants et 384 commis. C'était trop!

Source: Rapport diocésain: Centenaire de l'Eglise Catholique au Zaïre, 1989, p. 72. Diocèse de Buta: Evêché de Buta.

${ }^{24}$ KUYPERS et autres, op.cit., p. 29. 
Comme le fait observer le tableau ci-dessus, la rébellion a décimé presque tous les intellectuels de la sous-région soit $0,3 \%$ de la population totale des années 65-70. C'est d'ailleurs ce qui est resté impardonnable à l'endroit de Gbenye..

En guise de synthèse, disons (avec insistance) que ces dégâts matériels mais encore et surtout les massacres, assassinats anarchiques et incontrôlés, ont mis le Bas-Uélé à genoux. Le noyau intellectuel était vidé. L'économie s' était effondrée. L'Administration publique ne fonctionnait plus. La société était déstructurée. L'insécurité était totale. Tout le système dans sa globalité était secoué. Le tissu social de la sous-région avait perdu tout son fondement à cause des perturbations socio-économiques de la rébellion. Rien n'allait plus. Il a fallu la paix. Cette synthèse appelle un autre point qui concerne une période de reprise des activités.

\section{Le Bas-Uélé de 1966 à 1973}

Cette période fut caractérisée par une transition marquée d'une timide reprise des activités, avant de connaître une forte emprise de centralisation du pouvoir, elle-même suivie par la création du M.P.R. (Mouvement Populaire de la Révolution).

\section{a. La transition de 1966 à 1967}

Ainsi que nous l'avions dit, les pertes humaines et matérielles infligées par la rébellion avaient occasionné l'inertie et l'inactivité dans tous les domaines dans le district. Une pénible dissipation de l'impasse ne fut possible que vers le début de l'année 1966. En effet, quant le Haut-commandement militaire prit le pouvoir, la sous-région du Bas-Uélé était encore sous le joug des chefs rebelles et évoluait en vase-clos, le seul point de contact étant resté la RCA, par le Nord, et donc ouvert aux seuls Simba. Cette situation avait perduré jusquau mois de mars 1966, lorsque les opérations militaires, sous le commandement de Bob Denard, vinrent libérer la sous-région de l'emprise des Simba. ${ }^{25}$

\footnotetext{
${ }^{25}$ Témoignage de Frans Willems.
} 
Etant donné que les habitants avaient tous fui des agglomérations et centres pour se retrancher dans la brousse et parfois dans la foret profonde, le redémarrage des activités ne pouvait être envisageable qu'à certaines conditions. C'est pour cette raison qu'immédiatement après la délivrance de la sous-région et la reddition des Simba, la première préoccupation des autorités rétablies fut l'organisation de "rapatriement" des familles ayant survécu à la rébellion à leurs centres de travail, en l'occurrence Aketi, Buta, Bumba ou dans une certaine mesure Kisangani. Tous ces voyages avaient été réalisés grâce à la C.V.C. avec le concours de l'ANC qui assurait l'escorte.

Un trimestre entier était passé dans ces mouvements de rapatriement. Après seulement, les activités proprement dites devaient reprendre. Mais, disons sans tergiversation que la reprise a dû se faire dans de malheureuses conditions, et cela dans tous les secteurs. Cela est dû, comme nous l'avions déjà dit, au fait que les responsables et personnel radés étaient prèsque tous morts. Quelques amateurs vigilants devaient s'occuper du fonctionnement général des activités dont ils assuraient la mise en oeuvre. Ce fut donc une reprise vaille que vaille. Dans le domaine de l'enseignement par exemple, c'était le début de la catastrophe. Les enseignants étaient pour la plupart tués. Les quelques rescapés sortis des brousses étaient débordés. Le rendement avait été médiocre. Il a fallu attendre l'année 1969 pour que 1' Assistance Technique Generale - ATG vienne en rescousse.

Toujours à propos de la reprise des activités socio-économiques et culturelles, disons que le redémarrage n'avait pas encore donné ses effets lorsque les problèmes idéologiques sont venus étouffer les efforts. Il s'agit de la création du MPR en 1967.

\section{b. Le Bas-Uélé de 1967 à 1973: Rapprochement idéologique.}

La période qui va de 1967 à 1973 fut caractérisée par deux faits importants: les réformes administratives et la création du MPR (Mouvement Populaire de la Révolution).

En fait, les réformes administratives de 1967 ont déterminé le sort qui demeure celui des sous-régions rurales du Zaïre. Le décret de 1967 avait confirmé les 8 provinces, les 24 districts et les 132 territoires avec leurs chefferies et secteurs. Une forte hiérarchisation, obéissant à une forte centralisation du pouvoir de Kinshasa, était la seule caractéristique fonctionnelle des entités politico-administratives. A cet égard, les sous-régions lointaines, notamment le Bas-Uélé situé à plus de $2500 \mathrm{~km}$ fut exposé à une double situation que nous tentons d'aborder immédiatement. 
Cette complexe double situation s'exprime d'abord par la centralisation administrative, ensuite par la suppression de l'autonomie provinciale, et enfin par la pénétration du MPR dans les moindres contrées du pays. Tous ces faits sus-exprimés ont mis les sous-régions lointaines dans des situations très difficiles.

\section{$1^{\circ}$ Le Bas-Uélé face à la montée de l'emprise idéologique du MPR}

Le Bas-Uélé était encore dans sa phase de reprise des activités, ou les attaques sporadiques organisées par des Simba se manifestaient encore, or l'insécurité et la panique habitaient encore la population, que le M.P.R. naquit. Le pouvoir central avait placé la primauté du coté de l'idéologie, et pour s'implanter le parti a été placé à l'avant-plan. Les préoccupations politico-idéologiques ont supplanté celles des activités économiques. Cette situation n'a pas permis à la sous-région rurale du Bas-Uélé de progresser dans son cheminement de redressement économique. C'est dans cette logique de suprématie idéologique que le MPR fut institutionnalisé le 23 décembre $19700^{26}$ Une illustration simpliste peut marquer cette suprématie du M.P.R. Il s'agit du fait que partout à l'entrée de n'importe quel bureau, à la tête de toute correspondance officielle, il devait être obligatoirement mentionné en caractère gras: "MPR AVANT TOUT, LE RESTE IMMEDIATEMENT APRES". Cette inscription était devenue un slogan qui a pu remodeler la destinée de la population zaïroise. Cela voulait exprimer concrètement que lorsqu'une option était levée par le parti, le peuple, rassemblé de fait en son sein, ne devait que se mobiliser sans moindre contestation. Compte tenu de cette réalité, l'économie était reléguée au second plan par rapport et au profit de la politique.

La mobilisation politique était totale. L'encadrement idéologique était tel que le Bas-Uélé physiquement très éloigné de la capitale, était de plus en plus proche de celle-ci sur le plan idéologique et politique. Ce rapprochement politico-idéologique, renforcé par le manque du personnel adéquat dans la sous-région, a constitué un sérieux handicap au processus de redressement économique régulateur de la situation sociale. Nonobstant toutes ces difficultés, il y a eu tentatives de réorganisation d'activités économiques et culturelles.

\section{$2^{\circ}$ Effort de redressement}

Les entreprises qui avaient organisé la reprise des activités économiques dans le Bas-Uélé étaient la C.V.C., la Cotonco, la Comaco et dans une certaine mesure

\footnotetext{
${ }^{26}$ E.O. DJELO, Impact de la coutume sur l'exercice du ponvoir en Afrique: Cas du Zä̈re. Bel Elan LLN, 1990, p. 68.
} 
quelques entreprises privées. Quant aux activités socio-culturelles, la tâche était du côté de la territoriale mais surtout du côté des missions religieuses.

\section{a. La CVC}

Apres la rébellion, la Société des Chemins de Fer Vicinaux du Congo avait compté renouer avec toutes ses activités, notamment dans le domaine des transports. Le plus grand handicap de l'économie des régions rurales en particulier et du Zaïre en général reste l'inéluctable défi des transports. Dans le Bas-Uélé il y a eu et il y a encore aujourd'hui dégradation des infrastructures et vétusté des matériels. Le mal vient surtout de l'effondrement des transports terrestres par routes et rails. ${ }^{27}$ C'est pour pallier cette insuffisance que la CVC avait comme préoccupation la réfection des routes et la reconstruction des ponts détruits par les Simba. Pour remettre les trains sur les rails, la CVC se mit à retaper les anciennes locomotives. Après un dur labeur, les transports des biens et des personnes avaient repris, mais malheureusement pour une dure éphémère, car la zaïrianisation était venue de nouveau tout mettre en paille. Concrètement, les lignes Aketi-Komba-Bondo et Aketi-Buta-Andoma-Isiro-Mungbere avaient été ré-ouvertes et ont fonctionné normalement de 1968 à 1973 (même au-delà). Concernant les routes, tous les ponts avaient été réhabilités.

\section{b. La Cotonco}

Aussitôt que les mouvements de rapatriement avaient pris fin, la Compagnie Cotonnière du Congo (section Uélé) avait repris toutes ses activités. Les 15 usines du traitement de coton ont rouvert leurs portes. L'année 1968 avait connu une grande campagne du coton. Les cultivateurs ont fourni des milliers de tonnes de coton. Mais, comme pour la CVC, la Cotonco n'avait connu qu'une courte durée d'activités cotonnières.

\section{c. Les autres entreprises}

Ainsi que nous l'avions dit dans les pages précédentes, il s'agit des maisons de commerce tenues par des particuliers expatriés ou encore par quelques sociétés privées. Comme secteur d'activités, ces particuliers s'occupaient de l'agriculture, notamment des plantations de café, du caoutchouc, aussi de la commercialisation des denrées alimentaires et produits manufacturés. Sans trop nous répéter, nous présentons dans le tableau ci-dessous, les sociétés qui ont repris les activités économiques dans le Bas-Uélé.

\footnotetext{
${ }^{27}$ R. POURTIER, "Le Zaïre, un pays à reconstruire", Politique Africaine, Paris. 1990, p. 22.
} 
Ces quatorze maisons appartenant aux particuliers avaient renoué avec la commercialisation dans la sous-région or leurs établissements étaient éparpillés dans chaque zone et collectivité. Mais, nous n'avons pas les chiffres exacts de tous les magasins installés.

Pour clore ce chapitre, nous signalons que la COMUELE a repris très tard ses activités. Par ailleurs, signalons aussi que cette société a été rachetée tour à tour par plusieurs propriétaires avant d'être définitivement démantelée, dé-localisée au profit de CELZA dans la région de l'Equateur. Il y a eu également SOTRANSCOM et NOGUEIRA.

\section{Tableau $\mathbf{n}^{\circ} 2$}

\begin{tabular}{|l|l|l|l|l|}
\hline $\mathrm{N}^{\circ}$ & Etablissement & Propriétaire & Nationalité & Siège \\
\hline 1 & Magasin Kypri & Kyprianou & Grec & Buta \\
\hline 2 & Magasin Cyro & Cyro & Portugais & Buta \\
\hline 3 & Magasin Vassi & Vassilliou & Grec & Buta \\
\hline 4 & Trias & Taris & Grec & Akiti \\
\hline 5 & Dumtri & Dumtri & Grec & Buta \\
\hline 6 & Handi & Handida & Grec & Bondo \\
\hline 7 & Kyssorghis & - & "Juif" & Buta \\
\hline 8 & Ghgregrou & - & Grec & Buta \\
\hline 9 & Charolambo & - & "Juif" & Ango \\
\hline 10 & Thomaz & Thom & Portugais & Ango \\
\hline 11 & Makendo & - & Grec & \\
\hline 12 & Bambesa & & & \\
\hline 13 & Omari & Omari & Grec & Buta \\
\hline 14 & Lalji & Des indiens & Indien & Bondo \\
\hline 15 & Takis & Takis & Portugais & Poko \\
\hline
\end{tabular}

Source: Archives de la sous-région du Bas-Uélé, Buta, 1972, Section Agriculture et Commerce. 
d. Les efforts de redressement se sont également étendus sur le plan socio-culturel sous la conduite des confessions religieuses.

Outre l'oeuvre d'évangélisation les missions religieuses s'étaient évertuées à reprendre l'organisation des écoles selon les habitudes d'avant-rébellion. Dans l'ensemble le Bas-Uélé avait compté en 1972318 écoles primaires secondaires générales et professionnelles confondues avec 1022 classes pour une population totale de 515.048 habitants comme l'indique le tableau ci-après.

\section{Tableau n• 3}

\begin{tabular}{|l|l|l|l|l|l|l|l|}
\hline $\begin{array}{l}\text { Nomencla- } \\
\text { ture }\end{array}$ & Aketi & Ango & Bambesa & Bondo & Buta & Poko & TOTAL \\
\hline écoles & 56 & 49 & 43 & 47 & 63 & 60 & 318 \\
\hline classes & 225 & 906 & 176 & 201 & 248 & 226 & 1022 \\
\hline $\begin{array}{l}\text { Population } \\
\text { totale }\end{array}$ & 78.260 & 62.207 & 80.542 & 124.154 & 59.949 & 109.937 & 515.048 \\
\hline
\end{tabular}

Source: Archives du Bureau du Secrétariat de l'Enseignement Primaire et Secondaire. Service des Statistiques Scolaires, Effectifs globaux des écoles du Haut-Zaïre, 1976 à Kisangani.

Ces écoles avaient merveilleusement fonctionné jusqu'à la mesure d'étatisation décrétée en 1973 date à laquelle la gestion des écoles va connaître un début de dégradation qui n'a jamais cessé de s'amplifier.

Les principales congrégations et communautés à retenir sont:

Les Frères Maristes - Buta

Les Frères de Saint Gabriël - Bondo

Les Soeurs de St. Sépulcre - Aketi

La CBBU- Communauté Bapliste du Bas-Uélé - Buta (Bondo)

La CFP - Communauté des Fidèles Protestants - Bondo

L'UFM - Union des Fidèles et des Missionnaires - Aketi

Les Prémontrés - Aketi-Bambesa

Les soeurs Ursulines - Ango

En plus de ces écoles les missions religieuses s'étaient donné d'autres taches. De façon plus particulière les soeurs religieuses ont oeuvré pour la santé de la population dans toutes les formations sanitaires existantes dans le Bas-Uélé. 


\section{e. L'administration publique}

Subissant la hiérarchisation voulue par le MPR, les provinces étaient soumises à une forte centralisation et dépendaient de ce fait du pouvoir de la capitale. La stratégie des autorités du Parti entendait se servir de la territoriale pour s' allier la confiance de la population rurale (pour laquelle elles ne faisaient rien). Pour ce faire, le Pari avait ré-dynamisé et renforcé l'autorité des chefs coutumiers, chefs de cité, administrateurs territoriaux ${ }^{28}$, commissaires de districts. ${ }^{29}$ Leur tâche primordiale fut désormais d'inculquer aux administrés les idéaux du Parti. C'est pour cette raison que diverses réunions du Parti se tenaient selon un calendrier rigoureusement respecté. Dans le Bas-Uélé, le mercredi et le samedi se tenaient des séances d'animation politique. Les exécutants étaient recrutés parmi la population jeune et étaient ainsi détournés des activités agricoles. Les agents de la territoriale à quel niveau qu'ils soient étaient eux-mêmes soumis à des séances d'animation chaque matin avant de commencer les activités. De mauvaises habitudes furent installées. Au lieu de vaquer aux occupations quotidiennes, la population passait plus de temps à danser ou à participer à de longues réunions idéologiques stériles qui ne contribuaient qu'à affamer les gens.

Tel qu'on puisse le constater, ce rapprochement idéologique de la population du Bas-Uélé, n'a contribué qu'à l'enfoncer davantage dans son éloignement géographique.

\section{L'éloignement géographique du Bas-Uélé}

A titre de rappel, disons qu'à l'époque coloniale et durant les quatre années qui ont suivi l'indépendance du Zairre, le problème d'éloignement du Bas-Uélé n'était que psychologique. En réalité les moyens de transport et de communication fonctionnaient merveilleusement bien. Jusqu'en 1973, la CVC, la Cotonco et le TP avaient maintenu une parfaite régulation des communications dans le Bas-Uélé. Cependant lorsque les mesures de 1973 tombèrent, l'héritage matériel et l'infrastructure coloniale commençaient à devenir vétustes. Le délabrement s'installa et l'enclavement du Bas-Uélé devenait de plus en plus concret, car les routes étaient simplement devenues impraticables et les moyens de transport de plus en plus rares. Malgré la présence concrète du MPR, le Bas-Uélé a connu progressivement un enclavement quasi-total.

\footnotetext{
28 Actuels commissaires de zone.

${ }^{29}$ Actuels commissaires sous-régionaux.
} 
Telle est la situation qui a prévalu dans le Bas-Uélé de 1966 à 1973, un épisode marqué à la fois par un rapprochement idéologique entre villes et campagnes en même temps qu'un éloignement géographique entre ces dernières en république du Zaïre.

\section{Le Bas-Uélé de 1973 à 1985}

La coïncidence de trois événements phénoménaux survenus simultanément en 1973 ont conduit à la paupérisation des populations rurales du Zaïre en général, et de celle de la sous-région du Bas-Uélé en particulier. Il s'agit de la crise pétrolière, de la crise de croissance en Europe, de la zaïrianisation des biens des étrangers. ${ }^{30}$

Le troisième phénomène $\mathrm{a}$ agi de façon directe et déterminante dans la perturbation et la déstructuration de la vie socio-économique du Bas-Uélé. En fait, la zaïrianisation, décidée par les hommes politiques, avait pour source la philosophie politique dite I"'AUTHENTICITE". Elle fut matérialisée par une étatisation à outrance qui avait touché l'organisation économique du Bas-Uélé; elle avait déstabilisé les écoles, elle avait définitivement installé la misère du Bas-Uélé. La zairianisation était caractérisée d'abord par la confiscation des biens et entreprises des étrangers, ensuite par leur étatisation accompagnée d'une nationalisation mal pensée des responsables politiques, et enfin par la cession des biens confisqués aux acquéreurs, tous clients politiques du régime et non préparés en la matière. ${ }^{31}$ Il s'agit là de nouveau d'une impréparation comme ce fut le cas en 1960. Dans le Bas-Uélé, la zairianisation avait frappé la CVC, la Cotonco, les entreprises privées et les écoles.

\section{a. La CVC}

La nationalisation forcée et non préparée des cadres et la dé-localisation de la Direction générale de la CVC ont constitué le démantèlement de l' infrastructure de transport du Bas-Uélé. En effet, l'autorité politique qui redoute toujours une éventuelle sécession de la région du Shaba, voulait à tout prix s'attirer la confiance de cette région en vue de mieux la contrôler. C'est dans cette logique que toutes les sociétés des chemins de fer furent fusionnées en une seule: la SNCZ (Société Nationale des Chemins de Fer du Zaire) dont le siège social était placé à Lubumbashi. Cette fusion a occasionné une totale paralysie du secteur Nord or la CVC constituait l'épine dorsale des transports des Bas- et Haut-Uélés. Tous les matériaux avaient été transférés. Tous les cadres mutés. Pour les Uélés les transports des biens et des personnes furent arrêtés. Les conséquences de cette dé-localisation demeurent totales aujourdhui.

\footnotetext{
${ }^{30}$ TOENGAHO LOKUNDO, "Paradoxe des réformes territoriales au Zaïre", Zaïre-Afrique, $\mathrm{n}^{\circ}$ 251, Janvier 1991. CEPAS, Kinshasa, p. 11.

${ }^{31}$ E.O. DJELO, op.cit., p. 80.
} 


\section{b. La Cotonco}

Frappée de nationalisation, la Cotonco (et la Comaco) devenait l'ONAFITEX (Office National des Fibres et Textiles). Sa direction jadis installée à Dingela fut transférée d'abord à Kinshasa et ensuite à Kisangani sous la supervision de Soteski (Société Textile de Kisangani) une entreprise zaïro-française. Les matériaux furent simplement abandonnés, d'autres démantelés au profit de Soteski. Des lors la campagne de la production de coton avait cessé dans les Uélés et les cultivateurs délaissés jusque vers le début de l'année 1983. La pauvreté s'était réinstallée. Les zones durement frappées étaient Aketi, Bambesa, Bondo et Buta où les cultivateurs étaient de nouveau réduits en état de pauvreté.

\section{c. Les entreprises privées}

Ce sont les entreprises privées qui ont été les plus secouées par l'effet de zaïrianisation. Tous les particuliers auxquels nous avons fait allusion dans les pages précédentes (cfr. Tableau 2) ont été désemparés de leurs biens meubles et immeubles au profit des acquéreurs ${ }^{32}$ généralement peu formés, clients ou dignitaires du régime. La remise des biens se fit de façon anarchique de manière qu'au bout de trois ans seuls ceux dont les noms sont repris sur ce tableau avaient continué à fonctionner.

Tableau nº

\begin{tabular}{|l|l|l|l|l|}
\hline Noms & Métier & Designation & Secteur & Siège \\
\hline Bambule & Com.peuple & Plancom & Ag.com.transp & Likali-Aketi \\
\hline Kanalina & commis & Com Ding & “ & Barbesa-Buta \\
\hline Lekabusija & - & Ndjawe & agriculture & Buta-Poko \\
\hline Makala & Dipl.d'un & Atanaza & Agr.com & Buta-Likati \\
\hline Moboliaka & Enseignante & Mbobaka & Agr.com.trans & Bondo \\
\hline Mozagba & Com.Pr. & Entracibon & Com.ag.trans & Bondo \\
\hline Nendaka & Com.Pol. & Plan Kumu & “ & Gemeto-Poko \\
\hline
\end{tabular}

Source: Rapport de S/R, 1977, Buta.

${ }^{* *} \mathrm{~S} / \mathrm{R}=$ sous-région

${ }^{32}$ Désignés par moquerie acquis par erreur. 
A part ces huit personnes mentionnées, les autres acquéreurs étant entrés en possession des biens abondants sans en connaître l'origine, en ont disposé comme leur porte-monnaie. Sans notion ni principes de gestion économique des biens, ces acquéreurs ont fait faillite les uns après les autres, les stocks acquis ayant été vidés, les caisses trouées et tous les moyens matériels abîmés. Les magasins n'avaient plus que des rayons non achalandés. Le désastre fut total et la misère s'est installée. Une expression peut nous aider à vérifier le mécontentement de la population. En effet, tout le monde disait: "Tango ya Kala", "tango mindele bazalaka"...

Quant en ce qui concerne le café du Bas-Uélé, les choses devaient être tenues par des entreprises de l'Etat. A cet effet, il fut créé successivement l'OZACAF et l'ONC. Dans la sous-région on disait "Onase" pour désigner l'ONC. Ce qui voulait dire: "ce qui est à mente le sol", "ce qui ne vaut rien". Le découragement et le désarroi étaient totaux. Il a fallu attendre 1983 pour que la relève fut prise par d'autres sociétés.comme Plankumu, Plancom et Entracibon.

\section{d. Les écoles}

L'étatisation des écoles fut décrétée en 1973. Déjà vers la fin de l'année 1972, un différend avait affecté les relations entre le chef de l'Etat et le chef de l'Eglise Catholique Zaïrois. Cette situation a dégénéré en une mesure d'étatisation des écoles. Les missions religieuses cessaient d'être gestionnaires d'écoles. L'étatisation fut suivie d'une autre mesure. Il s'agissait de la radicalisation décrétée le 30 décembre 1974. Comme cela ne suffisait pas, une autre loi consacrait la rétrocession des écoles le 26 février 1977, matérialisée par une convention signée entre Etat et missionnaires en matière de gestion des écoles. ${ }^{34}$ Tous ces remue-ménage ont constitué un motif profond de défectuosité des écoles. Les passassions alternatives de l'organisation des écoles des gestionnaires en gestionnaires ont ruiné celles-ci. Déjà en 1973, quand les confessions religieuses avaient abandonné la gestion pour la première fois, les écoles ont connu une désorganisation notoire. Les mobiliers des écoles ont servi de meubles dans les maisons de nouveaux responsables. Les internats ont été pillés. Les subventions ont été détournées, etc. Les prévisions et provisions scolaires ne s'étaient plus faites durant les trois années qui ont suivi l'étatisation. Pour la première fois on s'était familiarisé à des expressions comme: "bulletin sauté" "bulletin zéro-zéro". Les manigances et les margoulinages de tous genres avaient

\footnotetext{
33 "Jadis", "quand les Blancs étaient là ... "

${ }^{34}$ A. DUA. Rapport de fin de stage des inspecteurs, IFCEPS, Kisangani, 1985, p. 45.
} 
élu domicile dans le chef de ceux qu'on appelle "agents paveurs". L'animation politique fut introduite dans les écoles. La pire des conséquences fut la dépravation des moeurs. L'école, avec l'étatisation, s'était détournée de sa loyale mission de formation civique. Comme autre méfait, ce fut la baisse du niveau d'enseignement de l'université à l'école de base.

\section{e. Les réformes de la Fonction Publique}

Les réformes de 1973 avaient touché l'administration et la fonction publique. C'est ainsi que la voirie et les travaux d'entretien des routes cessaient d'être du ressort de T.P. (Travaux Publics) et furent placés sous la direction de l'Office des Routes que les Zaïrois ont baptisé "Office des trous" à cause du mauvais fonctionnement. Cet office a plus contribué à la destruction plutôt qu'à la réfection et encore moins à la construction des routes. Même la transafricaine qui passe par Kisangani-Banalia-Buta-Bondo-Bangassou (RCA) n'est plus praticable. Le même scénario caractérise les tronçons Buta-Aketi-Bumba et Buta-Isiro. Comme l'on s'en rend compte, le Bas-Uélé est enclavé, il est éloigné, il n'a ni chemins de fer opérationnels, ni routes de dessertes agricoles. La misère demeure. La population croupit dans la paupérisation. Partout dans la sous-région les produits de plusieurs récoltes demeurent invendus, d'autres pourrissent. Les quelques kilos de café moisissent chez les planteurs. Le coton n'est plus planté, le paddy et l'arachide ne font plus objet d'aucune campagne. Tout cela à cause du fait qu'il n'y a pratiquement plus de routes.

En tout et pour tout, la zaïrianisation a inauguré une période catastrophique pour la sous-région du Bas-Uéle.

\section{Le Bas-Uélé vers les années 1980}

La campagne et la propagande électorales ayant précédé les élections de 1982 au Zaire avaient pour objectif la présentation des candidats qui devaient briguer des postes à trois niveaux différents, à savoir: le Commissariat politique; le Commissariat du peuple et les différents Conseils municipaux, urbains et locaux.

Cette campagne allait redonner espoir à la population du Bas-Uélé. En fait, les candidats devaient être élus dans leurs collectivités d'origine. Pour ce, ils étaient 
obligés de s'y rendre. Pour les six zones rurales qui constituent la sous-région du Bas-Uélé, la liste des candidats était très longue. Ayant tous été membres du MPR et de ce fait clients du régime, les candidats avaient tous bénéficié d'un crédit spécial, sauf ceux du niveau des Conseils locaux, pour faire leur campagne. C'est ainsi qu'ils ont du consentir d'énormes dépenses afin de s'assurer la confiance de l'électorat. Dans le Bas-Uélé les efforts de propagandes ont consisté notamment à relancer les activités de l'Office des Routes pour la réfection les routes abandonnées depuis l'année 1975. C'est en 1982 qu'on a revu des véhicules sillonner le Bas-Uélé. L'Office des Routes s'était remis au travail. Mais lorsque les élections eurent lieu, les heureux élus se retranchèrent pour la plupart dans la capitale.

Ayant bénéficié d'un gros financement, les candidats qui étaient aussi acquéreurs ont donné du tonus à leurs activités commerciales. Ce fut le cas du Plan Kumu de Nendaka et de Plancom de Bambule. Cependant nous devons signaler que les espoirs n'ont été qu'éphémères.

Un autre épisode très complexe à détenir reste l'exploitation artisanale de l'or dans la sous-région. Une ordonnance fut signée par le Président-Fondateur. ${ }^{35}$ Elle avait consacré la libéralisation de l'exploitation minière artisanale. ${ }^{36}$

En 1983 l'exploitation artisanale de l'or dans la zone de Bondo avait placé le BasUélé dans une autre vague. Il s'agit d'un détournement de vocation d'activités quotidiennes. Nous avions répété à maintes reprises que la population du Bas-Uélé est à vocation essentiellement agricole. Nous avons également dit que le manque de routes la non-existence du marché avaient découragé la population agricole. Trempée dans le bain d'une telle situation l'exploitation artisanale généralisée avait aggravé la misère.

D'après les enquêtes que nous avons personnellement menées pour la rédaction d'un mémoire de licence en 1988 nous avons constaté entre autres choses la désertion des écoles et par les élèves et par les enseignants; le versement de la population jeune dans le travail de l'or au détriment de l'agriculture. Sur le plan matrimonial le Bas-Uélé en général et les zones de Bondo Buta et Aketi en particulier ont connu des divorces des séparations de corps. Cette libéralisation de l'exploitation artisanale dans les multiples sites éparpillés dans la sous-région (cf. carte en annexe) n'a pas répondu aux ententes des autorités c'est-à-dire permettre aux filles et fils du pays de sortir de leur misère par les bénéfices qu'ils auraient tirés de l'exploitation artisanale de l'or. Au contraire cette activité a

\footnotetext{
${ }^{35}$ Ordonnance-loi ${ }^{\circ} 89 / 039$ du mois de novembre 1982

${ }^{36}$ Vundu awe Ta Penako, "Décentralisation territoriale des responsabilités au Zaire, Pourquoi et Comment'?", in Zaïre-Afrique, n 166 , juin 1982.
} 
conduit à l'abandon des activités agricoles. Une illustration malheureuse reste qu'entre 1984 et 1986 le riz consommé dans le Bas-Uélé venait de Kisangani. Les travaux de l'or ont contribué à consolider la misère de la population plutôt qu'à la rayer. Les formations hospitalières ont été désertées l'Administration publique abandonnée etc. Que de malheureux constats!

Un fait pourrait paraitre paradoxal il s'agit du fait que plusieurs sociétés étaient venues implanter des comptoirs d'achats de l'or à Buta et à Bondo. D'autres sont entrées jusqu'à Baye $\left(80 \mathrm{~km}\right.$ de Bondo soit $600 \mathrm{~km}$ de Kisangani) ${ }^{37}$ Monsieur Dokolo $^{38}$ avait même installé un Bac à Gangu pour la traversée des véhicules qui alimentaient les différentes carrières de l'or constituées au-delà de Baye. Les grands comptoirs non pirates étaient: SCIBE ZAÏRE, MAYATA, COGEK et KILO-MOTO.

Concrètement la période comprise entre 1983-1986 a connu un trafic fréquent entre Kisangani et les sites d'exploitation de l'or. Mais l'impact de ce trafic $\mathrm{n}^{\mathrm{\prime}}$ a pas été positif pour la sous-région.

\footnotetext{
${ }^{37}$ D.S., MILY, op cit., p. 83.

${ }^{38}$ DOKOLO: un des grands hommes d'affaires et propriétaires du Zaire, fondateur de la Banque de Kinshasa. B.K.
} 


\section{NOTICE BIBLIOGRAPHIQUE}

\section{A. Ouvrage principaux}

CARBONELLE \& KIRSCHEM, L'économie de deux Uélés, Bruxelles, 1961.

CHALUX, Un an au Congo belge, Albert Dewit, Bruxelles, 1925.

COMELIAU, C.,Fonctions économiques et pouvoir politique. La province de l'Uélé en 1963-64, Collection études économiques, nº 1, République du Congo, 1964.

C.V.C., Congo-Nil, Van Sassche, Bruxelles, 1936.

DENIS et WILMET, L'Afrique Centrale et Orientale, PUF, Paris, 1971.

DJELO, E.D., Impact de la coutume sur l'exercice du pouvoir en Afrique. Cas du Zaïre, B.E. LLN, 1990.

GONIDEC, E.P.C., Série Afrique: le Zaïre, Berger Levrault, Paris, 1973.

KAMA, Fizi, Géographie du Zaïre, Paris Hatier, 1970.

TSHIMANGA wa TSHIBANGU, Histoire du Zaire, Ceruki, Bukavu, 1969.

VERHAEGEN, B., Rébellion au Congo. Maniema, Tome II, CRISP, 1969.

VERHOEVEN, J., Dagboek, Gijzelaars over Makondo, Diest, 1965.

\section{B. D'autres documents}

\section{Revues}

DE ST. MOULIN, L., "Organisation administrative du Congo de 1888 à 1992", Zä̈re Afrique, $\mathrm{n}^{\circ} 261$, janvier 1992, CEPAS, Kinshasa.

GONDO, A.I., "Chiffres de populations, les élections législatives de 1987", Zaïre Afrique, $\mathrm{n}^{\circ} 164$, avril 1988, CEPAS, Kinshasa.

POURTIER, R., "Le Zaïre, un pays à reconstruire", Politique africaine, Paris, 1990. 
TOENGAHO, L., "Paradoxe des réformes territoriales au Zaïre",Zaïre-Afrique, $\mathrm{n}^{\circ} 251$, janvier 1961, CEPAS, Kinshasa.

VUNDU AWE TE PEMAKO, "La décentralisation territoriale des responsabilités au Zaïre. Pourquoi, comment?", Zaire-Afrique, $\mathrm{n}^{\circ} 166$, juin 1982, CEPAS, Kinshasa.

\section{Mémoire}

MILY DENDA-SAKALA, D. , Libéralisation de l'exploitation artisanale de l'or et la scolarisation à Bondo. (1983-1987), Mémoire de licence, 1988, IPN, Kinshasa, Inédit.

\section{Rapport}

DUA Alipade, Rapport de fin de stage des inspecteurs d'enseignement, I.F.C.E.P.S., Kisangani, 1985.39 